\title{
Anomalous scaling in a non local growth model in the Kardar-Parisi-Zhang universality class
}

\author{
Mario Castro ${ }^{1}$, Rodolfo Cuerno ${ }^{2}$, Angel Sánchez ${ }^{2}$, and Francisco Domínguez-Adame ${ }^{1}$ \\ ${ }^{1}$ GISC, Departamento de Física de Materiales \\ Facultad de Ciencias Físicas, Universidad Complutense, E-28040 Madrid, Spain \\ ${ }^{2}$ GISC, Departamento de Matemáticas \\ Escuela Politécnica Superior, Universidad Carlos III de Madrid, E-28911 Leganés, Madrid, Spain
}

\begin{abstract}
We study the interface dynamics of a discrete model previously shown [A. Sánchez, M. J. Bernal, and J. M. Riveiro, Phys. Rev. E 50, R2427 (1994)] to quantitatively describe electrochemical deposition experiments. The model allows for a finite density of biased random walkers which irreversibly stick onto a substrate. There is no surface diffusion. Extensive numerical simulations indicate that the interface dynamics is unstable at early times, but asymptotically displays the scaling of the Kardar-Parisi-Zhang universality class. During the time interval in which the surface is unstable, its power spectrum is anomalous; hence the behaviors at length scales smaller than or comparable with the system size are described by different roughness exponents. These results are expected to apply to a wide range of electrochemical deposition experiments.
\end{abstract}

The field of non equilibrium dynamics of rough surfaces and interfaces has undergone an enormous activity during the last decade. Considerable experimental and theoretical efforts have been devoted to understanding the common general features of the growth of surfaces in various seemingly unrelated phenomena [1,2]. By exploiting the existence of universality and scale invariance akin to those present in equilibrium critical phenomena, most of the activity has been aimed to organizing non equilibrium surface growth processes into universality classes. In this context, surfaces are described by the global width (or roughness) $W(L, t)$, the rms fluctuations of the height variable $h(x, t)$ giving the location of the surface at position $x$ over a substrate of lateral size $L$ at time $t$, around its mean value $\bar{h}_{L}(t)=\frac{1}{L} \sum_{x} h(x, t)$ :

$$
W^{2}(L, t)=\frac{1}{L}\left\langle\sum_{x}\left(h(x, t)-\bar{h}_{L}(t)\right)^{2}\right\rangle,
$$

where angular brackets stand for noise average.

In many cases it is observed that the width initially grows as $W(L, t) \sim t^{\beta}$ for $t \ll L^{z}$, saturating at a size dependent value $W(L) \sim L^{\alpha}$ for $t \gg L^{z}$. The roughness exponent $\alpha$, the dynamic exponent $z$ and their ratio $\beta=\alpha / z$ are usually taken to identify the universality class the growth process considered belongs to. Under the assumption of scale invariance (self-affinity), analogous behavior is expected for the rms fluctuations of the height within a box of lateral size $l \ll L$, or local width

$$
w^{2}(l, t)=\frac{1}{l}\left\langle\sum_{x}\left(h(x, t)-\bar{h}_{l}(t)\right)^{2}\right\rangle,
$$

namely

$$
w(l, t) \sim \begin{cases}t^{\beta} & \text { if } t \ll l^{z} \\ l^{\alpha} & \text { if } t \gg l^{z}\end{cases}
$$

The scaling behavior (3) holds, e.g., in the Kardar-ParisiZhang (KPZ) equation [3], which generically describes surfaces growing irreversibly in the absence of specific conservation laws [2]. However, in recent studies [4,5] of surface growth in the context of Molecular Beam Epitaxy (MBE) and related vapor deposition techniques, it has been found that many relevant discrete models and continuum equations do not obey the local scaling (3), but rather for intermediate times $l^{z} \ll t \ll L^{z}$ it is found that $w(l, t) \sim l^{\alpha_{l o c}} t^{\left(\alpha-\alpha_{l o c}\right) / z}$, with $\alpha_{l o c} \neq \alpha$; hence the behaviors of the surface at small and large length scales differ. This phenomenon has been termed anomalous scaling, and is not yet understood at a fundamental level (see [6,7] for overviews). In principle, anomalous scaling was somehow associated with super-roughening (i.e., the existence of exponent values $\alpha \geq 1$ ). However, recent works have shown that it can appear as an independent phenomenon [9] (henceforth referred to as intrinsic anomalous scaling) caused by anomalous behavior of the surface power spectrum [4, 10] (to be defined below). Up to now, the only cases in which anomalous scaling has been identified are related to models with a conserved current along the surface, as those for $\mathrm{MBE}$ growth [6.7], or with some type of disorder 8 12]. We are aware of no previous report on the occurrence of intrinsic anomalous scaling in models with time dependent noise and no constraint or conservation law. Moreover, an obviously important open issue is to clarify the experimental conditions under which anomalous scaling can be observable.

In this Rapid Communication we study a surface growth model, called multiparticle biased diffusion limited aggregation (MBDLA), introduced in 13 and 
shown to describe quantitatively electrochemical deposition (ECD) experiments 14. In particular, it allows to study systems with a finite density of depositing particles of different species. The relaxation rules of MBDLA do not yield a conserved current. Specifically, consistent with a range of experimental applications [14], there is no surface diffusion. As a consequence, we will show below that MBDLA asymptotically displays the scaling behavior of the KPZ universality class. Nevertheless, there does exist a time regime in the evolution of the surface during which the effective scaling is intrinsically anomalous and described by non universal exponents. This time regime is associated with the onset of an instability due to the Laplacian character of the model. This feature is analogous to recent reports 15 that for a class of model discretized equations in which there exists a conserved current, anomalous scaling with non universal exponents is present during a transient regime in which the surface growth is unstable. Here we find an analogous phenomenon for a realistic model without conserved currents which belongs to the KPZ universality class. Actually, our findings are reminiscent of a discrete non conserved erosion model [16] also displaying an initial instability followed by asymptotic KPZ scaling. The crucial difference is that the model in 16] is described by the noisy Kuramoto-Sivashinsky equation, asymptotically equivalent to KPZ in 1+1 dimensions [7], and - as KPZ itselffree of anomalous scaling.

The algorithm that defines MBDLA begins with a number of random walkers (cations) randomly distributed with concentration $c$ on a two-dimensional square lattice with periodic boundary conditions. The lower side of the lattice is chosen to be the cathode. The initial condition evolves in time as follows: Every time step a walker is chosen and moved to one of its four neighboring sites with different probabilities: 0.5 to move parallel to the cathode (either left or right); $0.25+p$ to move down towards the aggregate (the cathode, at time $t=0$ ) and $0.25-p$ to move up, away from it. The parameter $p$ is referred to as the bias and, as shown in [13], can be quantitatively related to the electric current in the physical system; experimentally, it can be controlled by changing the value of the applied electric field. After a destination site has been selected, the particle moves if that node of the lattice is empty (and we select another particle if it is not). Once the particle has been moved, if the new position has any nearest neighbor site belonging to the aggregate, the walker's present position is added to the aggregate with probability $s$; otherwise it stays there (and is able to move again) with probability $1-s$. We term $s$ the sticking probability; it is related to the chemical activation energy the cation needs to stick to the aggregate.

Of the two parameters of MBDLA, the bias $p$ and the sticking probability $s$, only $p$ is independent for the experimental description, $s$ being simply proportional to it
[13. This remark notwithstanding, in this work we will consider both $p$ and $s$ as independent parameters, for our goal is to make a general analysis of the model behavior beyond the experimentally motivated values. As seen in what follows, this will help to obtain a coherent, complete picture of MBDLA properties. Thus, $s$ takes values between 0 and 1 , and $p$ ranges from 0 to 0.25. Finally, let us note that MBDLA produces in general a non compact aggregate which can have voids and overhangs. Large values of $p$ produce nearly ballistic trayectories, while smaller values drive the particles in a Brownian motion fashion. In two limits, the behavior of MBDLA approaches other relevant models: ballistic deposition (for $p=0.25$ ), well known to belong to the KPZ universality class and not to display anomalous scaling [2], and multiparticle Diffusion Limited Aggregation (MDLA) (for $p=0$ ), the paradigmatic model of unstable Laplacian growth [1].

To begin the summary of our results, we will describe the phenomenology for $p=0.05$ and arbitrary $s$. Physically, $p=0.05$ represents not too small applied electric fields, surface diffusion being negligible, so that MBDLA provides a good description of ECD. We will later discuss how changes in $p$ modify the description that follows. Simulations were performed in a $L=300$ by 1500 lattice, although other values of $L$ were also considered (see below). The dynamics of the model is conveniently characterized by the global width, Eq. (11).

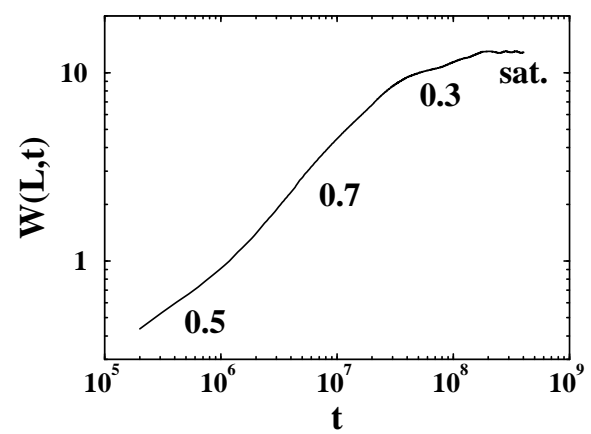

FIG. 1. Global width vs time for $p=0.05, s=1, c=0.1$. The dynamics presents an initial transient during which the surface is still uncorrelated and $\beta \sim \frac{1}{2}$. Then there is an unstable transient within which $\beta \sim 0.7$. In this region the system presents intrinsic anomalous scaling (see text). Before saturation, the KPZ nonlinearity governs the dynamics, hence $\beta \sim 0.33$. The present plot is an average over 50 noise realizations. All magnitudes are measured in arbitrary units.

We are interested in the dynamics of the active zone [1], hence we define the variable $h(x, t)$ to be the height of the topmost particle belonging to the cluster for each column. As mentioned above, for times before saturation due to finite system size $\left(t<L^{z}\right)$, the width is expected to scale as $W(L, t) \sim t^{\beta}$. In our case, we find that, along the time evolution, $\beta$ takes up to three different values for large enough systems, see Fig. 1. At early 
times, shot noise dominates the growth, thus $\beta \sim 0.5$ as in a random deposition process. As time proceeds, the Laplacian character of the model shows up, favoring the unstable development of pillars, which in turn leave deep grooves behind. This instability is reflected in the large value of the growth exponent $(\beta \sim 0.59-0.73$ increasing with sticking probabilities). As local slopes of pillars (or grooves) become larger, they seem to trigger further nonlinear effects, whose consequence is the stabilization of the surface dynamics. Thus, for $s \gtrsim 0.5$ we measure in this regime $\beta \sim 0.25$, a value close to that of the Edwards-Wilkinson universality class [18. For smaller values of the sticking probability, $s \lesssim 0.5$, we measure $\beta \sim 0.33$, the growth exponent characteristic of the KPZ universality class. Actually simulations for larger system sizes $(L=512,1024)$ yield $\beta \sim 0.33$ before saturation for almost all values of $s$. Hence the asymptotic behavior of the model is described by KPZ scaling. This also shows the role of $s$ as a noise reduction parameter 17, large noise reduction $(s \rightarrow 0)$ tuning the system closer to the asymptotic scaling regime. The value of the roughness exponents are $\alpha_{\text {loc }} \sim \alpha \sim 0.5$ in all cases, showing that there is no anomalous scaling in the asymptotic state. Measurements of the mean excess velocity $v(m)$ of an interface subject to an average tilt $m$, imposed through helical boundary conditions, confirm [19 the relevance of the KPZ nonlinearity in the effective description of our system. Once established that the long time scaling behavior of the interface is of the KPZ type, we focus on the unstable transient regime, characterized by two main features, namely a large $\beta$ value (hence very rapid growth) and intrinsic anomalous scaling caused by a non standard form of the power spectrum of the surface, $S(k, t)=\langle\widehat{h}(k, t) \widehat{h}(-k, t)\rangle$, where $\widehat{h}(k, t)=L^{-1 / 2} \sum_{x}\left[h(x, t)-\bar{h}_{L}(t)\right] \exp (\mathrm{i} k x)$.

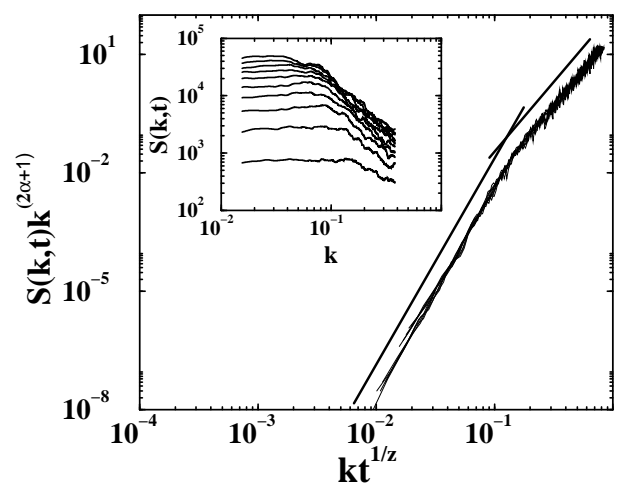

FIG. 2. Collapsed power spectrum within the unstable transient using the same parameters as in Fig. 1. The inset shows the time evolution of the power spectrum every $10^{6}$ time units. Thick lines have slopes given by the exponents quoted in Table I. All magnitudes are measured in arbitrary units.

In Fig. 2 we plot the colapse of the power spectrum $S(k, t)$ within the unstable transient, where the inset shows $S(k, t)$ as function of $\mathrm{k}$ for every $10^{6}$ time units. $S(k, t)$ displays a behavior consistent with the scaling form

$$
S(k, t)=k^{-(2 \alpha+1)} s\left(k t^{1 / z}\right),
$$

where

$$
s(u)= \begin{cases}u^{2 \theta} & \text { if } u \gg 1, \\ u^{2 \alpha+1} & \text { if } u \ll 1 .\end{cases}
$$

The exponent $\theta=\alpha-\alpha_{l o c}$ measures the difference between the global roughness exponent $\alpha$ and the local roughness exponent $\alpha_{\text {loc }}$. The exponents are $\alpha \sim 2.15$, $\alpha_{\text {loc }} \sim 0.5, \beta \sim 0.73$, and $z \sim 2.95$ (all exponent values given are to within an accuracy less than $5 \%$ ). It has been shown in Ref. [10] that a structure factor such as (4) implies intrinsic anomalous scaling for the local width. The slopes of the collapsed curves yield exponents consistent with the ones used to achieve the best collapse. Collapses of structure factor data obtained for different $s$ values yield similar qualitative results, as shown in Fig. 3, and the exponent values summarized in Table I. All the exponent values (except notably $\alpha_{l o c}$, see also [6, 9, 10]) depend strongly on model parameters, and we consider them as non universal effective values.

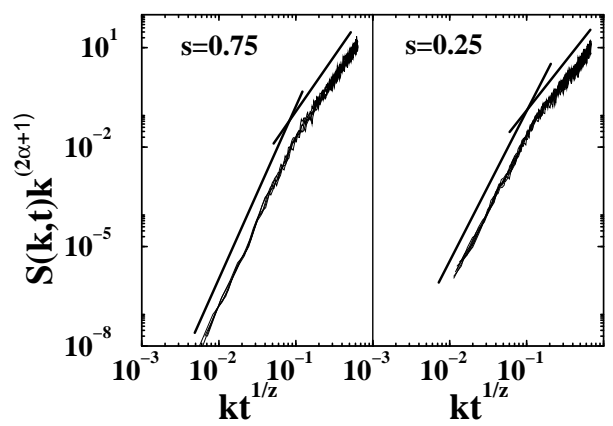

FIG. 3. Collapsed power spectra within the unstable transient using the exponent values in Table I, with $s=0.75$ and $s=0.25$. Parameters other than $s$ are as in Fig. 1. Thick lines have slopes given by the exponents quoted in Table I. All magnitudes are measured in arbitrary units.

To provide a general description of our model, we have studied bias values different from $p=0.05$. For example, for $p=0.005$ and large values of the sticking probability $s$, we have observed DLA like patterns as expected [13]. Consequently the unstable regime lasts longer than in the cases studied above. The same is observed for larger values of $s$ at low $p$ values. Moreover, decreasing (increasing) the ion concentration $c$ increases (decreases) the duration of the unstable growth, in accordance with the fact that as $c \rightarrow 0$ pure DLA is recovered. Hence we can tune the two parameters and the concentration to reproduce different experimental behaviors, because changes in the parameters alter the relative duration of 
the different scaling regions in the dynamics of the system. However the asymptotic KPZ scaling still holds at long enough times.

In summary, we have shown the existence of intrinsic anomalous scaling for a realistic model, MBDLA, without conserved currents and hence in the KPZ universality class. Anomalous scaling appears within a time region of variable duration (depending on parameter values) in which the surface evolves in an unstable fashion. This type of scaling is due to a non-standard form of the power spectrum and features non-universal (parameter dependent) values of the effective exponents. We interpret anomalous scaling to be associated with the unstable structures developing in the model dynamics. Thus, for large length scales we measure $\alpha>1$, associated with the steep slopes of pillars. On the other hand, when probing short length scales we obtain $\alpha_{l o c} \sim 0.5$ (see Table I), a value numerically close both to the KPZ exponent and to the exponent resulting from the convolution of the Heaviside function with the interface [21] (through our definition of the height variable $h(x, t))$. At any rate, intrinsic anomalous scaling seems to be strongly associated in our case with the nonlocality of the evolution rules of the model which produces the unstable structures of the interface.

To date, anomalous scaling has been mostly observed in theoretical models, hence the interest of our results: as MBDLA is a correct description of ECD [14], our findings could be observed in that kind of experiments. Interestingly, MBDLA displays instabilities similar to those reported in various ECD experiments [20], reinforcing the connection of the model to actual physical systems. Moreover, anomalous scaling may explain the wide spread in the $\alpha$ values $(\alpha \sim 0.5-0.8)$ reported in various ECD experiments [20]. As shown in [9], in the presence of anomalous scaling, the fact that all length scales saturate at the same global saturation time $t_{\text {sat }} \sim L^{z}$ poses additional difficulties to the evaluation of exponents by means of the local width. Specifically, if not performed at global saturation, the roughness exponent thus obtained is an effective value different from both, $\alpha$ and $\alpha_{l o c}$. Research on extensions of the model to more experimental parameters is under way, the influence of surface diffusion being the center of our future work.

\section{ACKNOWLEDGMENTS}

We are indebted to Enrique Diez for his participation in the early stages of this work. We thank Miguel Angel Rodríguez, Miguel Angel Rubio, Javier Buceta and Ricardo Brito for helpful comments. This work has been partially supported by CICyT (Spain) under Project No. MAT95-0325.
* Electronic address: mario@valbuena.fis.ucm.es

[1] T. Vicsek, Fractal Growth Phenomena, 2nd. edition (World Scientific, Singapore, 1992).

[2] A.-L. Barabási and H. E. Stanley, Fractal Concepts in Surface Growth (Cambridge University, Cambridge, 1995).

[3] M. Kardar, G. Parisi, and Y.-C. Zhang, Phys. Rev. Lett. 56, 889 (1986).

[4] M. Schroeder, M. Siegert, D. E. Wolf, J. D. Shore, and M. Plischke, Europhys. Lett. 24, 563 (1993).

[5] J. Amar, P.-M. Lam, and F. Family, Phys. Rev. E 47, 3242 (1993); S. Das Sarma, S. V. Ghaisas, and J. M. Kim, ibid. 49, 122 (1994).

[6] S. Das Sarma, C. J. Lanczycki, R. Kotlyar, and S. V. Ghaisas, Phys. Rev. E 53, 359 (1996).

[7] J. Krug, Adv. Phys. 46, 139 (1997).

[8] L. A. N. Amaral, A.-L. Barabási, H. A. Makse, and H. E. Stanley, Phys. Rev. E 52, 4087 (1995).

[9] J. M. López and M. A. Rodríguez, Phys. Rev. E 54, R2189 (1996).

[10] J. M. López, M. A. Rodríguez, and R. Cuerno, Phys. Rev. E 56, 3993 (1997); Physica A 246, 329 (1997).

[11] M. Jost and K. D. Usadel, Phys. Rev. B 54, 9314 (1996); J. M. López and M. A. Rodríguez, J. de Physique I (France) 7, 1191 (1997).

[12] For a recent report of anomalous scaling in fracture surfaces, see J. M. López and J. Schmittbuhl (to appear).

[13] A. Sánchez, M. J. Bernal, and J. M. Riveiro, Phys. Rev. E 50, R2427 (1994).

[14] J. M. Riveiro and M. J. Bernal, J. Non-Cryst. Solids 160, 18 (1993).

[15] C. Dasgupta, J. M. Kim, M. Dutta, and S. Das Sarma, Phys. Rev. E 55, 2235 (1996).

[16] R. Cuerno, H. A. Makse, S. Tomassone, S. T. Harrington, and H. E. Stanley, Phys. Rev. Lett. 75, 4464 (1995); K. B. Lauritsen, R. Cuerno, and H. A. Makse, Phys. Rev. E 54, 3577 (1996).

[17] J. Kertész and D. E. Wolf, J. Phys. A 21, 747 (1988).

[18] S. F. Edwards and D. R. Wilkinson, Proc. R. Soc. London A 381, 17 (1982).

[19] We have obtained the parabolic shape $v(m)=v(0)+\frac{\lambda}{2} m^{2}$ characteristic of a KPZ nonlinear term, see J. Krug and H. Spohn, Phys. Rev. Lett. 64, 2332 (1990).

[20] G. L. M. K. S. Kahanda, X. Zou, R. Farrell, and P. Wong, Phys. Rev. Lett. 68, 3741 (1992); J. M. Pastor and M. A. Rubio, Phys. Rev. Lett 76, 1848 (1996).

[21] P. Keblinski, Phys. Rev. Lett. 71, 805 (1993); G. L. M. K. S. Kahanda, P. Wong, Phys. Rev. Lett. 71, 806 (1993).

\begin{tabular}{|c|c|c|c|c|}
\hline \hline$s$ & $\beta$ & $\alpha$ & $\alpha_{\text {loc }}$ & $z$ \\
\hline \hline 1.00 & 0.73 & 2.15 & 0.50 & 2.95 \\
\hline 0.75 & 0.67 & 2.04 & 0.49 & 3.03 \\
\hline 0.50 & 0.67 & 1.92 & 0.54 & 2.87 \\
\hline 0.25 & 0.59 & 1.73 & 0.50 & 2.94 \\
\hline \hline
\end{tabular}

TABLE I. Summary of relevant exponents as measured with $S(k, t)$ and $w(l, t)$ within the unstable transient, for $p=0.05, s=1, c=0.1$. 